\title{
ANATOMÍA Y MORFOLOGÍA DE ÓRGANOS REPRODUCTIVOS DE Nephelium lappaceum $\mathrm{L}$.
}

\author{
Jesús Reyes-Moreno ${ }^{1}$, Eduardo García-Villanueva ${ }^{1}$, Manuel Reyes-Moreno ${ }^{1}$ \\ y Jesús M. E. Aguilar-Luna²
}

\begin{abstract}
RESUMEN
El fruto del rambután (Nephelium lappaceum L.) es exótico, no tradicional y muy atractivo en los mercados internacionales; sin embargo, la información sobre sus aspectos anatómicos es escasa. El objetivo del estudio fue describir la anatomía de iniciación y diferenciación floral, desarrollo de gametofitos, del arilo y tipo de fruto de $N$. lappaceum. Se implementó un diseño longitudinal que consistió en detectar los cambios anatómicos de las yemas vegetativas hacia las reproductivas, el desarrollo de las flores pistiladas, así como del ovario y óvulo de la flor hermafrodita, hacia el fruto y semilla. La iniciación floral se presentó a los 41 días después de preantesis. El estambre tiene una antera con dos tecas, las anteras son indehiscentes y presentan epidermis, el endotecio desarrolla engrosamientos fibrosos, tiene células binucleadas y el tapete es de tipo secretor. Se apreció un lóculo por teca, con granos de polen triporados. La microsporogenesis se desarrolló normalmente, la división de las células madres de las micrósporas fue simultánea y las tétradas de las micrósporas, con disposición tetraédrica, se encontraban encerradas por el tapete que es bastante delgado. El gineceo presenta un ovario bicarpelar o tricarpelar. Cada lóculo contiene un óvulo campilótropo, bitegumentado y crasinucelado; el megagametofito es de tipo polygonum. El fruto alcanzó su máximo tamaño, peso y color a los 125 días después de antesis; es seco, indehiscente, monospermo; deriva de un carpelo y es una nuez esquizocárpica.
\end{abstract}

Palabras-clave adicionales: Esporofito, esquizocárpico, floración, fructificación, gametofito

\begin{abstract}
Anatomy and morphology of reproductive organs of Nephelium lappaceum $\mathbf{L}$

The rambutan fruit (Nephelium lappaceum L.) is exotic, non-traditional and very attractive in international markets; however, currently there is little information on anatomical and morphological aspects. Therefore, the objective of this study is to describe the anatomy of floral initiation and differentiation, development of gametophytes, of the aril and type of the fruit of $N$. lappaceum. A longitudinal design was implemented that comprises in the detection of anatomical changes of the vegetative buds towards the reproductive ones; development of pistillate flowers, as well as the ovary and ovule of the hermaphrodite flower, towards the fruit and seed. The floral initiation was presented after 41 days of pre-anthesis. The stamen has an anther with two thecae, the anthers are indehiscent and have epidermis, the endothecium develops fibrillar thickenings, it has binucleate cells and the tapetum is secretory. A locule per theca appeared with triporate pollen grains. Microsporogenesis developed normally, division of mother cells of the microspores was simultaneous and the tetrads of the microspores, with tetrahedral arrangement, were enclosed by the tapetum, which is quite thin. The gynoecium shows a bicarpellary or tricarpellary ovary. Each locule contains a campylotropous, bitegmic and crassinucellate ovule; the megagametophyte is of polygonum type. The fruit reached its maximum size, weight, and color 125 days after anthesis. It is dry, indehiscent, monospermo; it is derived from a carpel and is a schizocarpic nut.
\end{abstract}

Additional keywords: Flowering, fruiting, gametophyte, schizocarpic, sporophyte

\section{INTRODUCCIÓN}

El rambután (Nephelium lappaceum L., Sapindaceae), es un frutal exótico originario del sureste de Asia que se desarrolla en regiones tropicales húmedas; sus rendimientos van de 9,81 $\mathrm{Mg} \cdot \mathrm{ha}^{-1}$ (México), hasta $15 \mathrm{Mg} \cdot \mathrm{ha}^{-1}$ (Malasia). Es una fruta atractiva que se consume en fresco, con un alto valor en el mercado de países de Europa y América del Norte (Joo et al., 2017).

Sus flores son zigomorfas o casi actinomorfas homoclamideas, blanquecinas, de 2 a $4 \mathrm{~mm}$ de longitud y $3 \mathrm{~cm}$ de ancho; pueden ser masculinas y/o hermafroditas (Lim, 1984; Windarsih y Efendi,

Aceptado: Enero 4, 2021

Recibido: Mayo 4, 2020

y Productividad. Estado de México, México.

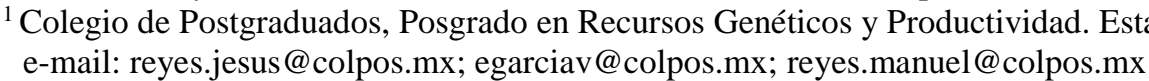

${ }^{2}$ Benemérita Universidad Autónoma de Puebla, Complejo Regional Norte, Programa de Ingeniería Agroforestal. Puebla, México. e-mail: mao.aguilar@correo.buap.mx (autor de correspondencia) 
2019). Si en estas últimas las anteras no abren, la flor funciona como pistilada, y si los estigmas no abren funciona como estaminada. El cáliz consta de 4 a 6 sépalos verde-amarillentos, de 1 a $2 \mathrm{~mm}$ de largo; no desarrollan la corola. Emergen en las axilas del eje terciario de las inflorescencias con crecimiento parcial tipo dicasio (Stern y Gazit, 2003). La flor estaminada posee de 5 a 8 estambres de 1,5 a $3 \mathrm{~mm}$ de longitud, anteras dehiscentes y gineceo reducido a un pistilo rudimentario (González et al., 2014). La flor pistilada presenta estambres de $1 \mathrm{~mm}$ de longitud, anteras indehiscentes; gineceo sincárpico con 2 a 3 carpelos, estilo filiforme y estigma bífido, número limitado de óvulos (por lo general sólo uno o dos) en cada lóculo (Lim, 1984). La presencia de un disco nectario entre el androceo y el gineceo es una adaptación a su polinización entomófila (Nacif et al., 2001).

El óvulo presenta de cinco a siete estratos celulares en el lado de la antirafe. El micrópilo está delimitado por ambos tegumentos y conformado por células isodiamétricas en hileras de 40 a 70 estratos de grosor (Lim, 1984; Johri et al., 2014). La cálaza está formada por células de paredes delgadas con color marrón. El óvulo es campilótropo (con nucela curva) o bien casi anátropo y es crasinucelado, es decir, donde la célula madre de la megaspora y el desarrollo subsecuente de la misma se encuentran separados de la epidermis por una o más capas de células, por lo que se encuentra profundamente embebido dentro del tejido esporofítico (Barrel y Grossniklaus, 2005). El ovario es súpero, trilocular y los óvulos de placentación axial; está cubierto de tricomas glandulares que desaparecen del fruto con la madurez (Lim, 1984), tal como sucede con otras sapindáceas como Handeliodendron bodinieri (H.Lev.) Rehder (Cao et al., 2008) y Delavaya toxocarpa Franch (Li y Nian, 2009).

Según Kothagoda y Rao (2012), el fruto de $N$. lappaceum puede ser drupa o baya; el pericarpio tiene de 2 a $4 \mathrm{~mm}$ de grosor y está recubierta con 200 a 300 estructuras suaves, puntiagudas y alargadas conocidas anatómicamente como espiternos o tricomas (de 10 a $12 \mathrm{~mm}$ de longitud según el cultivar); es rojo, amarillo o anaranjado (Caballero et al., 2011). El arilo (crecimiento especializado del funículo de la semilla) constituye la porción comestible más importante, es fácilmente removido de la semilla y tiene una apariencia traslúcida con sabor agridulce (Joo et al., 2017; Arévalo et al., 2018). El fruto después de la cosecha cambia su aspecto, pues va perdiendo humedad y oscureciéndose rápidamente (Reyes et al., 2020).

Para este frutal no se encuentran descritas las etapas de desarrollo que dan origen a la formación del fruto; de la misma forma existe controversia respecto de si se trata de una drupa o una baya (Cao et al., 2008; Kothagoda y Rao, 2012). Por lo anterior, el objetivo del presente estudio fue describir la anatomía de la iniciación y diferenciación floral, desarrollo de gametofitos, del arilo y tipo de fruto de $N$. lappaceum.

\section{MATERIALES Y MÉTODOS}

La investigación se realizó en una huerta comercial de $N$. lappaceum 'See-Chompoo' de cinco años de edad, ubicada en Frontera Hidalgo, Chiapas, México (14\%44' N, 92 $\left.10^{\circ} \mathrm{W}, 46 \mathrm{msnm}\right)$. El clima es cálido subhúmedo, con régimen de lluvias en verano (García, 2004). Durante el estudio se instaló un datalogger digital que registró una temperatura promedio de $27,7^{\circ} \mathrm{C}$, y promedios de HR máxima de $68,4 \%$ y HR mínima de $46,5 \%$. El suelo es franco arenoso, con 5,75 de pH, 4,92\% de MO y fertilidad media.

El marco de plantación es de 7 × 6 m entre hileras y árboles; en la huerta se aplicó fertirrigación, excepto del 1 de noviembre al 31 de diciembre de 2015, para inducir la floración. Se trabajó con 18 árboles (de cinco años de edad) elegidos aleatoriamente (cada árbol representó una unidad experimental). En ellos se hicieron 18 muestreos que iniciaron cuatro meses antes de la floración, recolectando ápices de yemas, flores y frutos de la parte media del árbol, y finalizaron en el mes de mayo, abarcando nueve etapas de desarrollo del cultivo (Cuadro 1).

Se implementó un diseño longitudinal que consistió en detectar los cambios anatómicos de las yemas vegetativas hacia las reproductivas, así como del ovario y óvulo de las flores hermafroditas que funcionan como pistiladas, hacia el fruto y la semilla; recolectando tres muestras de cada etapa fenológica por árbol. Para lo cual, el material vegetal se subdividió en secciones de 0,5 cm y fijó en FAA (formaldehido, ácido acético, etanol 70 \%) en una relación 5:5:90; después, el material se deshidrató en etanol para 
hacerlo más firme (Sandoval, 2005). Para lograr su infiltración con parafina histológica, se siguió el protocolo establecido por Shields y Heinbockel (2018). Posteriormente, se realizaron cortes de $15 \mu \mathrm{m}$ con micrótomo rotatorio y se colocaron en el portaobjetos con adhesivo de cromo (Sandoval, 2005). Se realizó el desparafinado e hidratación, y finalmente la tinción con safranina verde rápido y el montaje en bálsamo de Canadá (Haseloff, 2003). Las secciones se observaron y analizaron con un microscopio Tetraview LCD Digital Touch Screen. Las preparaciones se realizaron para cada unidad experimental y por fecha de muestreo. El desarrollo de los frutos se observó directamente en cada caso, desde el prendimiento hasta la maduración.

Cuadro 1. Muestreos para el estudio fenológico-estructural de $N$. lappaceum

\begin{tabular}{|c|c|c|c|c|c|}
\hline $\begin{array}{l}\text { Fecha de } \\
\text { muestreo }\end{array}$ & $\begin{array}{c}\text { Material } \\
\text { vegetal }\end{array}$ & $\begin{array}{l}\text { Etapa de } \\
\text { desarrollo }\end{array}$ & $\begin{array}{l}\text { Fecha de } \\
\text { muestreo }\end{array}$ & $\begin{array}{c}\text { Material } \\
\text { vegetal }\end{array}$ & $\begin{array}{c}\text { Etapa de } \\
\text { desarrollo }\end{array}$ \\
\hline 1 oct 2015 & Yema vegetativa & Periodo inductivo & 15 ene 2016 & Botón flor. & Diferenciación floral \\
\hline 10 oct 2015 & Yema vegetativa & Periodo inductivo & 20 ene 2016 & Flor & Floración plena (antesis) \\
\hline 20 oct 2015 & Yema vegetativa & Periodo inductivo & 5 feb 2016 & Flor & Postantesis \\
\hline 5 nov 2015 & Yema vegetativa & Periodo inductivo & 15 feb 2016 & Fruto & Desarrollo estado 1 \\
\hline 15 nov 2015 & Yema vegetativa & Periodo inductivo & 1 mar 2016 & Fruto & Desarrollo estado 2 \\
\hline 30 nov 2015 & Yema vegetativa & Iniciación floral & 1 abr 2016 & Fruto & Llenado de fruto \\
\hline $10 \operatorname{dic} 2015$ & Yema vegetativa & Diferenciación floral & 25 abr 2016 & Fruto & Llenado de fruto \\
\hline 20 dic 2015 & Yema vegetativa & Diferenciación floral & 10 may 2016 & Fruto & Madurez de fruto (cambio de color) \\
\hline $31 \operatorname{dic} 2015$ & Yema vegetativa & Diferenciación floral & 25 may 2016 & Fruto & Madurez de fruto (cambio de color) \\
\hline
\end{tabular}

\section{RESULTADOS Y DISCUSIÓN}

El proceso de iniciación floral se presentó a los 41 días después de preantesis (DDP), lo cual se comprobó con el cambio anatómico de las yemas axilares ubicadas en el lado inferior inmediato a la inflorescencia reproductiva del ciclo anterior (Figura 1a, 1b y 1c), apreciándose con una elongación, un engrosamiento y un aplanamiento del meristemo apical de una yema axilar (Figuras 1d y 1e), que culminó con la reorganización anatómica del meristemo y la aparición verticilada de los primeros antófilos. A los 21 DDP el $100 \%$ de las yemas recolectadas se encontraron completamente diferenciadas, con la aparición del meristemo apical de la inflorescencia (Figuras 1f y $1 \mathrm{~g}$ ), apreciándose los primordios de los hipsófilos y sus respectivas yemas axilares, las cuales dieron origen a las flores.

El escapo floral se originó del meristemo apical de una yema axilar, con entrenudos cortos o largos. En sus nudos se insertan los hipsófilos de cuyas yemas axilares se forman las ramificaciones de segundo orden y de las yemas axilares de sus hipsófilos se forman las flores. La ramificación de primer orden se reduce gradualmente a lo largo del eje y hasta el ápice, donde se forma el último dicasio apical. La inflorescencia es tipo panícula con crecimiento parcial o dicasio; es frecuente encontrar los dos tipos de flores en la misma inflorescencia.

En el presente experimento la inducción floral pudo deberse al estrés hídrico al que fueron sometidos los árboles durante dos meses, más que a la temperatura, ya que en esta última no hubo variación considerable $\left(27,2 \pm 2,2{ }^{\circ} \mathrm{C}\right)$. Menzel (2001) reportó que árboles de Litchi chinensis Sonn requieren un periodo de temperaturas bajas $\left(<10{ }^{\circ} \mathrm{C}\right)$ para la inducción floral; de igual forma se infiere que las condiciones podrían ser similares para Dimocarpus longan Lour, el cual posee un fruto con semillas que presentan arilos comestibles y cuya maduración en el sureste asiático se presenta en la misma época del año que para $L$. chinensis (Pham et al., 2015). Siendo $N$. lappaceum una sapindácea, se esperaría un estímulo similar; sin embargo, esto no pudo comprobarse debido a que la oscilación térmica durante el estudio no propició bajas temperaturas. Entonces, la inducción floral parece estar relacionada con un periodo de estrés hídrico, idea reforzada con el estudio realizado en Colombia por Arias et al. (2016) en N. lappaceum.

Hacia la madurez del gineceo de las flores pistiladas se desarrolló sólo el ovario de un carpelo y el otro quedó rudimentario (abortivo) (Figura 2); a este fenómeno se le identificó como una separación carpelar postgénita como consecuencia del desarrollo del fruto.

Las estructuras en los cortes anatómicos 
realizados coinciden con las observaciones efectuadas por Nacif et al. (2001), quienes mencionaron que el ovario en la flor de $L$. chinensis presenta dos lóbulos y un septo muy desarrollado, el cual divide al ovario en dos lóculos; así, cada lóculo contiene un óvulo, anátropo, bitegumentado
(Endress, 2011). En N. lappaceum se desarrolló sólo un lóbulo del ovario, este último bicarpelar o tricarpelar con placentación axial (Figuras 3a y 3b), mientras que el otro detuvo su crecimiento, permaneciendo rudimentario en el pedúnculo; tal como fue descrito por Lim (1984).

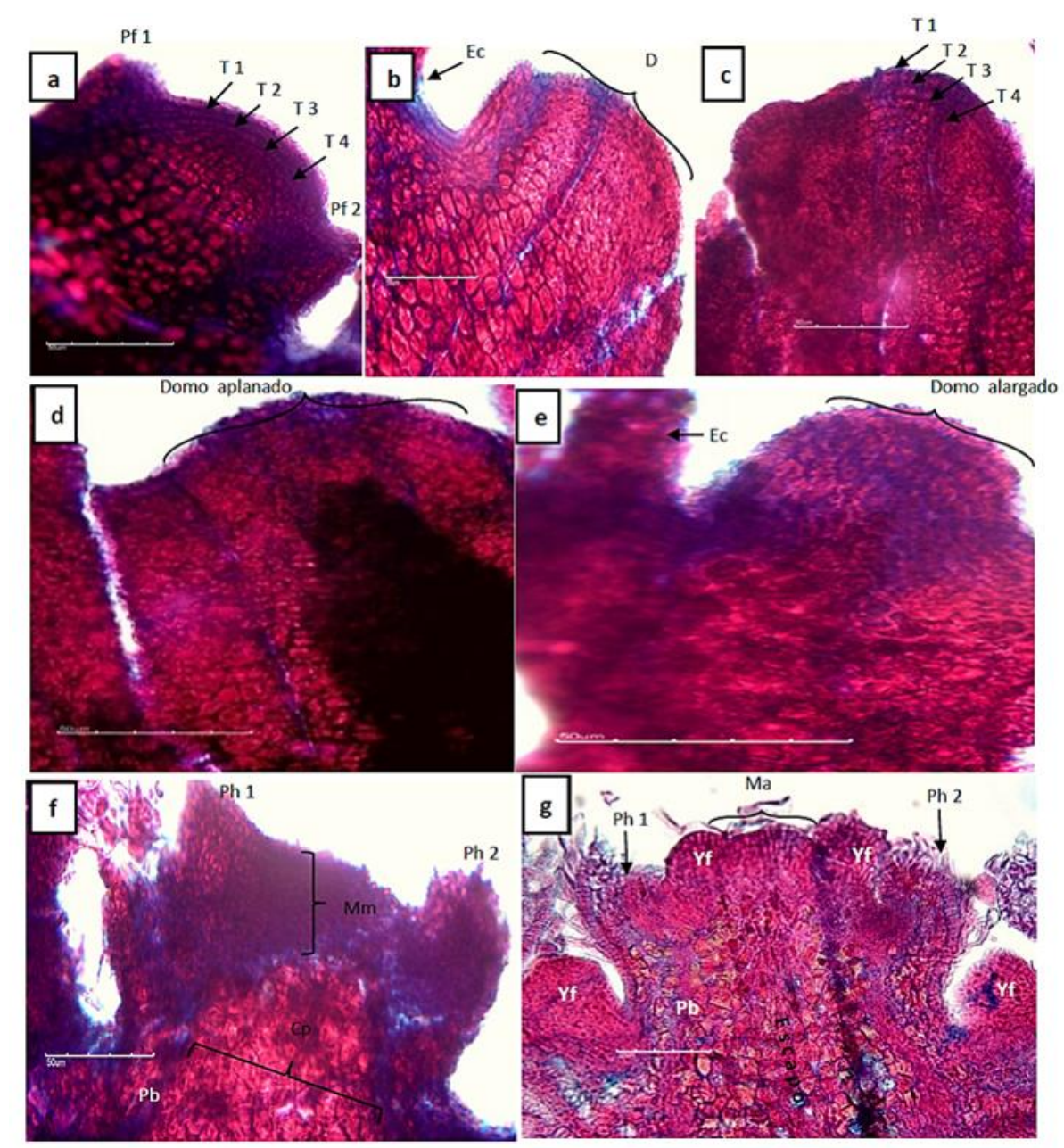

Figura 1. Proceso de diferenciación floral en $N$. lappaceum, cortes longitudinales en: a) yema vegetativa a los 116 DDP; b) yema vegetativa a los 96 DDP; c) yema vegetativa a los 66 DDP; d) domo aplanado a los 52 DDP; e) domo alargado (iniciación) a los 41 DDP; f) meristemo en diferenciación a los 31 DDP; g) meristemo apical de la inflorescencia a los 21 DDP. Cp: parénquima central, D: domo, Ec: escama, Ma: meristemo apical, Mm: manto meristemático con cuatro estratos celulares, Pf: primordio floral, $\mathrm{Pb}$ : procámbium, Ph: primordio foliar, T: túnica, Yf: yema floral. Escala: $50 \mu \mathrm{m}$

La microsporogénesis, observada durante la antesis, se desarrolló normalmente, con producción de cuatro microsporas haploides; a partir del muestreo del 20 de enero y en los cortes anatómicos, el estambre mostró una antera con dos tecas y un lóculo por teca. Las anteras, indehiscentes, presentan epidermis y células binucleadas; la antera de $N$. lappaceum presentó cuatro microsporangios (Figura 4a), con un filamento bien desarrollado en el cual se encontró un haz vascular. Los microsporangios se encontraron rodeados por el tapete, el cual es delgado, secretor y consta de un estrato celular con paredes delgadas (Figura 4b). Esta observación coincide con el estudio realizado por Zini et al. (2012) en Melicoccus lepidopetalus Radlk, donde 
la pared de la antera estuvo formada por la epidermis, la cual es poco desarrollada; y consiste del endotecio, dos estratos parenqui-máticos intermedios y el tapete (Figura 4c).

La flor mostró cuatro microsporangios, con división simultánea de las células madre de micrósporas, encerradas por el tapete y endotecio con engrosamiento fibroso (Figura 4b), lo cual coincide con lo reportado por González et al. (2014) en L. chinensis, donde el endotecio en esta fase se encuentra engrosado, formado por células rectangulares y tangencialmente angostas. De igual forma, Zini et al. (2012) mencionaron que en $M$. lepidopetalus el endotecio presentó un engrosamiento fibrilar y en la antesis las micrósporas fueron unicelulares.

El inicio de la microgametogénesis se manifestó poco antes de la liberación de los granos de polen (dehiscencia de la antera), al formarse la exina ornamentada y al abrirse la antera para liberarlos (Figura 4c). En esta etapa los granos de polen son gametofitos jóvenes o inmaduros, pues aún no han formado los gametos masculinos o células espermáticas mediante la mitosis de la célula generativa (Skinner y Sundaresan, 2018). El grano de polen en $N$. lappaceum es triporado, similar a la observación de González et al. (2014) en Allophylus edulis (A. St.- Hil.) Radlk. ex Warm (Sapindaceae). El polen consiste de dos células, una grande (vegetativa) y en su interior otra pequeña con doble membrana (generativa), esta última con su propio núcleo mientras que la vegetativa presentó membrana simple con doble pared celular (intina y exina).

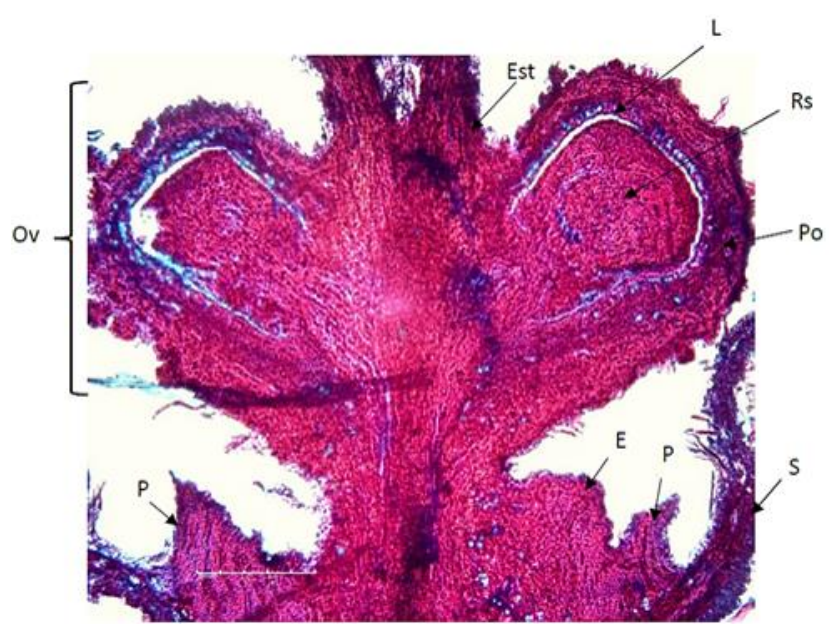

Figura 2. Gineceo de flores pistiladas de $N$. lappaceum en corte longitudinal. Floración plena (antesis). E: estambres, Est: estilo, L: lóculo, Ov: ovario, P: pétalos, Po: pared del ovario, Rs: rudimento seminal, S: sépalos. Escala: $50 \mu \mathrm{m}$
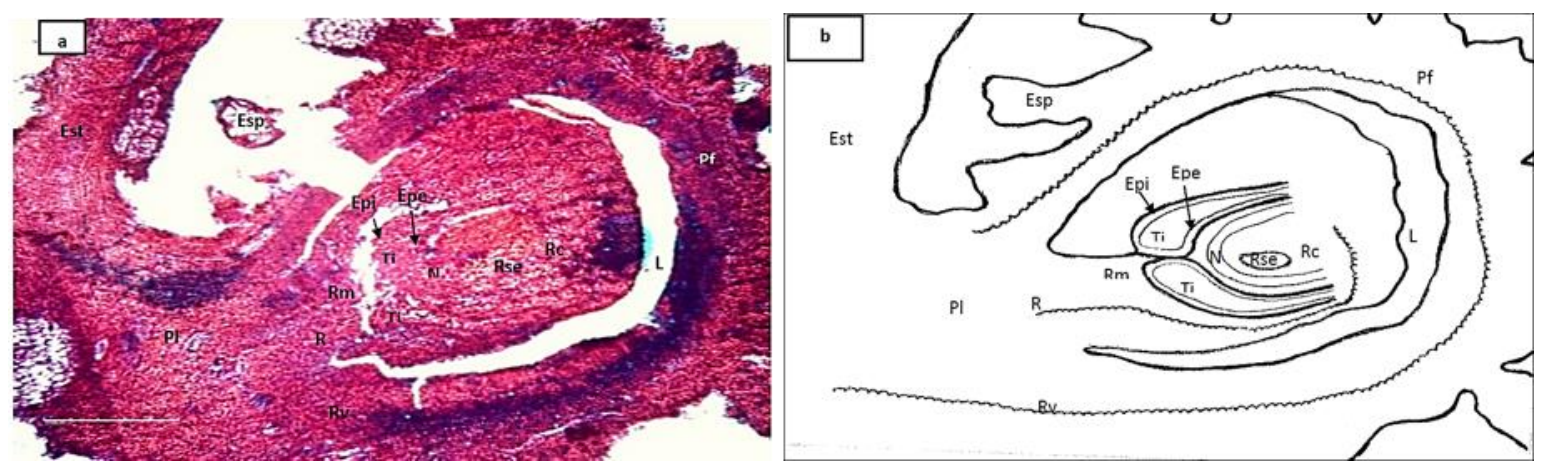

Figura 3. Anatomía del ovario en $N$. lappaceum a los 16 días después de antesis. a) corte longitudinal; b) representación esquemática. Epe: epidermis externa, Epi: epidermis interna, Esp: espiternos, Est: estilo, L: lóculo, N: nucela, Pf: pared del fruto, Pl: placenta, R: rafe, Rc: región calazal, Rm: región micropilar, Rse: región del saco embrionario, Rv: región vascular, Ti: tegumento interno. Escala: $50 \mu \mathrm{m}$ 

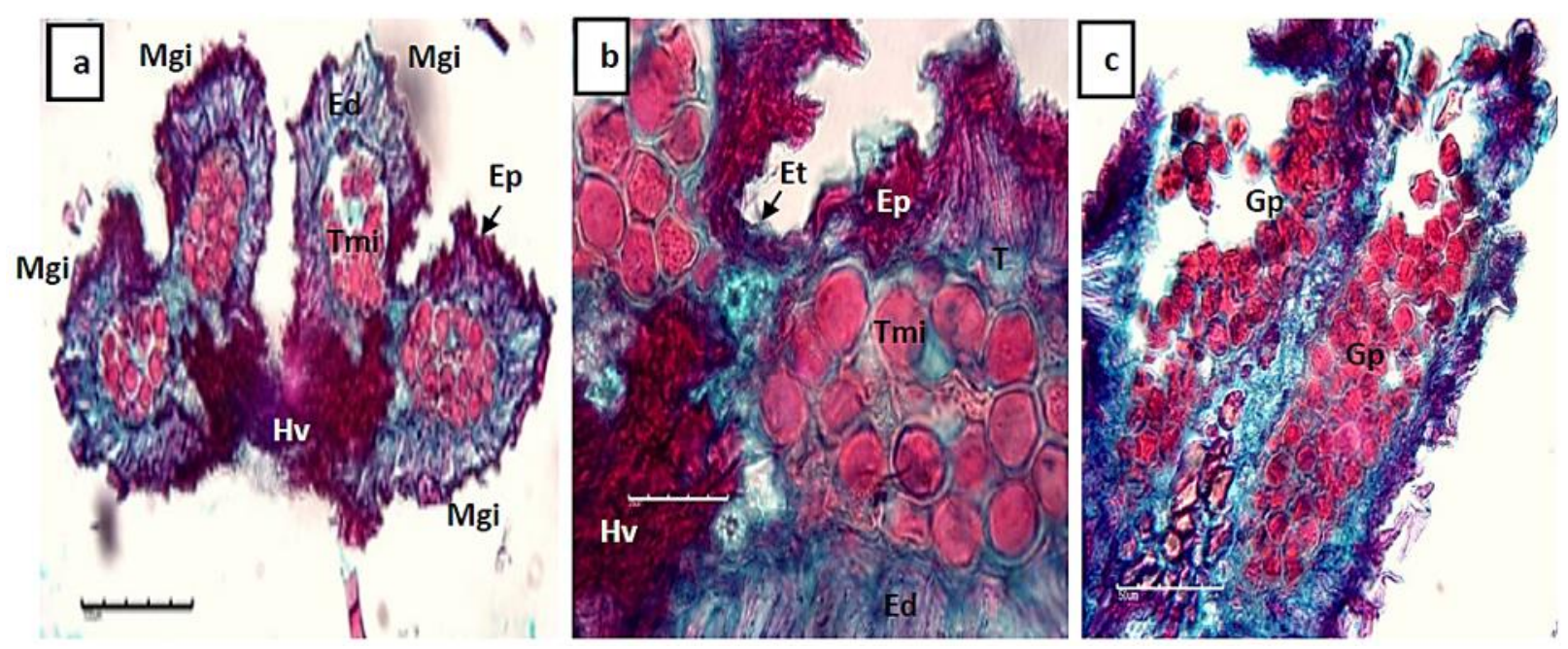

Figura 4. Antera de flor estaminada en antesis de N. lappaceum en: a) plano longitudinal antera donde se observan cuatro microsporangios; b) endotecio en plano transversal; c) antera dehiscente con granos de polen. Ed: endotecio, Ep: epidermis, Et: estomio, Gp: granos de polen, Hv: haz vascular, Mgi: microsporangio, T: tapete, Tmi: tétradas de microsporas. Escala: $a=50 \mu \mathrm{m}, \mathrm{b}=100$ $\mu \mathrm{m}, \mathrm{c}=50 \mu \mathrm{m}$

En postantesis, a la caída de los sépalos, a los 16 días después de antesis (DDA), se encontraron restos de estilo en el gineceo. En el óvulo campilótropo, bitegumentado y crasinucelado se observó la región del saco embrionario. La nucela del óvulo es el megasporangio y es aquí donde se diferencia la célula madre de la megaspora. Según Lim (1984), durante la megasporogénesis, esta célula madre (diploide) experimenta meiosis dando lugar a cuatro células haploides. Se observó que el óvulo presenta parénquima nucelar, epidermis nucelar multiestraficada, epidermis interna, externa $\mathrm{y}$ parénquima interepidérmico del tegumento interno; así como epidermis interna, externa y parénquima interepidérmico del tegumento externo, región micropilar y calazal del saco embrionario, placenta, lóculo y pared del fruto el cual tuvo origen en la madurez al pericarpio (Figura 5).
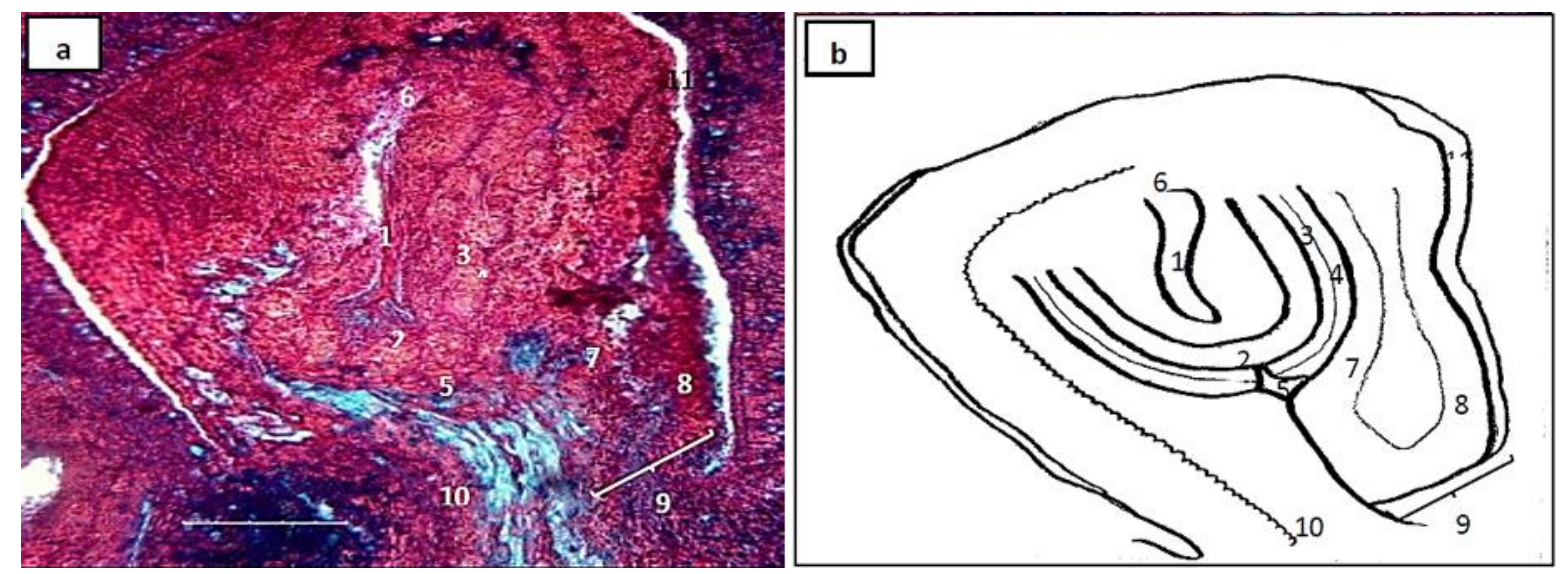

Figura 5. Anatomía y estructura del óvulo en $N$. lappaceum a los 16 DDA. a) sección longitudinal; b) representación esquemática. 1: región del saco embrionario, 2: nucela, 3: epidermis interna del tegumento interno, 4: epidermis externa del tegumento interno, 5: región micropilar, 6: región calazal, 7: epidermis interna del tegumento externo, 8: epidermis externa del tegumento externo, 9: tegumento externo, 10: rafe, 11: lóculo. Escala: $50 \mu \mathrm{m}$ 
Se observó que, durante la megagametogénesis, la megaspora funcional dio lugar al gametofito femenino maduro. En este sentido, Yadegari y Drews (2004) afirman que la megaspora funcional sufre una o más divisiones mitóticas sin citocinesis resultando en un coenocito multinucleado. Posteriormente, las paredes celulares son formadas alrededor de esos núcleos, resultando en un saco embrionario maduro. En $N$. lappaceum, la megagametogénesis o formación del saco embrionario monospórico es de tipo polygonum, de la megaspora calazal, con cuatro núcleos micropilares y cuatro calazales.

El megagametofito queda conformado por siete células: la ovocélula, dos sinérgidas (haploides), la célula central (gametofíticamente diploide) y tres antípodas (haploides). El óvulo se encontró unido a la placenta del ovario por un apéndice denominado funículo (el obturador no fue observado claramente, pero se asume cerca de la región del micrópilo) como sucede en A. edulis (Gonzalez et al., 2014) y otras especies (Endress, 2011).

Así, el desarrollo de la pared del fruto se observó a los 16 DDA, aunque esto difiere del estudio de Nacif et al. (2001), quienes mencionaron que el pericarpio se desarrolla intensamente a partir de los 7 DDA, y hacia el final se encontraron los espiternos en desarrollo. A los 26 DDA, sólo se observó al embrión y al endospermo en desarrollo (Figura 6 a y 6 b). El origen de las estructuras que conforman la semilla y el fruto después de las dos singamias se presentan en el Cuadro 2.

Inicialmente, los derivados del megasporangio que participan en el desarrollo de la semilla son tanto del parénquima, como de su epidermis; los demás darán origen a las estructuras anatómicas que formarán parte de la cubierta seminal: testa y tegmen. En el primer estado fenológico (frutos en desarrollo, estado 1), se observaron restos de nucela y desarrollo de su pared.También se observó el inicio de la endospermogénesis (Figura 6b), donde la célula madre del endospermo comenzó a dividirse antes que el cigoto, el cual se asume es triploide (3n, producto de la fusión del núcleo de la célula central con el de la segunda célula espermática) y presenta un tipo de desarrollo nuclear normal, debido a que la célula madre se dividió varias veces, formando posteriormente la nucela y las células tegumentarias. El arilo inició su desarrollo aproximadamente a los 70 DDA e inició su crecimiento desde el funículo de la semilla, cerca de la región micropilar hasta que este tejido cubrió la totalidad de la semilla en la madurez del fruto (Figura 7A).

En una sapindácea similar (D. longan), Pham et al. (2015) mencionaron que el desarrollo del fruto consta de dos etapas principales: inicio del del pericarpio (de 1 a 50 DDA), y desarrollo del embrión. A este respecto, los resultados difieren de lo encontrado en $N$. lappaceum, ya que el desarrollo del pericarpio se observó antes del desarrollo del arilo. En la semilla joven, a los 25 DDA se observaron restos de endospermo, el cual se redujo hacia la madurez por el embrión (Figuras 7B y 8C); en este sentido, el endospermo se forma, pero se degrada antes de que se suscite el inicio del proceso de germinación.

El obturador, aunque no observado, se asume que es persistente en la región micropilar y aparece como una membrana delgada en media luna alrededor de la cara abaxial de la parte superior del funículo, tal como es descrito en otras sapindáceas (Nacif et al., 2001; Weckerle y Rutishauser, 2005). El endospermo presentó un tipo de desarrollo nuclear, en el cual las divisiones estuvieron acompañadas del desarrollo de paredes celulares que aíslan células uninucleadas o plurinucleadas (Baroux et al., 2002).

En este estado fenológico de la semilla inmadura aún no se encontró el arilo, debido a que este no se formó sino hasta 70 DDA. La semilla madura es ovoide y el embrión está conformado por la plúmula, nudo cotiledonal y radícula (Figura 8A y 8B); hacia la madurez fue arilada $y$ exalbuminosa; sus dimensiones promedio sin arilo son: $9,80 \pm 0,49 \mathrm{~mm}$ de largo por 7,20 $\pm 0,50 \mathrm{~mm}$ de ancho y 6,10 $\pm 0,63 \mathrm{~mm}$ de grosor, con un peso promedio de 4,5 $\pm 0,87 \mathrm{~g}$. La testa fue mucho más estratificada que el tegmen y su tejido vascular no fue prominente en el rafe (Johri et al., 2014).

Asimismo, se encontró el tegumento interno del cual se formó el tegmen y el tegumento externo del cual se formó la testa hacia la madurez; el tegumento interno del óvulo se originó por las divisiones periclinales de las células dérmicas y el tegumento externo se desarrolló de la epidermis y del tejido subepidérmico (Endress, 2011). En $N$. lappaceum el tegumento externo es más grueso que el interno (Figura 6a y 6c), y esta observación difiere de lo registrado por González et al. (2014) en $A$. edulis (Sapindaceae), donde el tegumento interno se desarrolló más que el externo. También se observó que el rafe entra hasta la región calazal 
(región del óvulo que se localiza entre el hilo o funículo y la calaza) (Figura 7B).

El fruto alcanzó su tamaño máximo, peso y color a los 125 DDA, periodo que coincide con lo descrito para N. lappaceum (Arévalo et al., 2018) y para D. longan (Pham et al., 2015), quienes mencionaron que los frutos presentan un periodo de crecimiento de cuatro meses después de antesis. El fruto midió en promedio $4,5 \mathrm{~cm}$ de longitud y hasta $4 \mathrm{~cm}$ ancho, lo que le confiere una forma ovoide, con crecimiento de tipo sigmoidal simple (Muhamed et al., 2019).
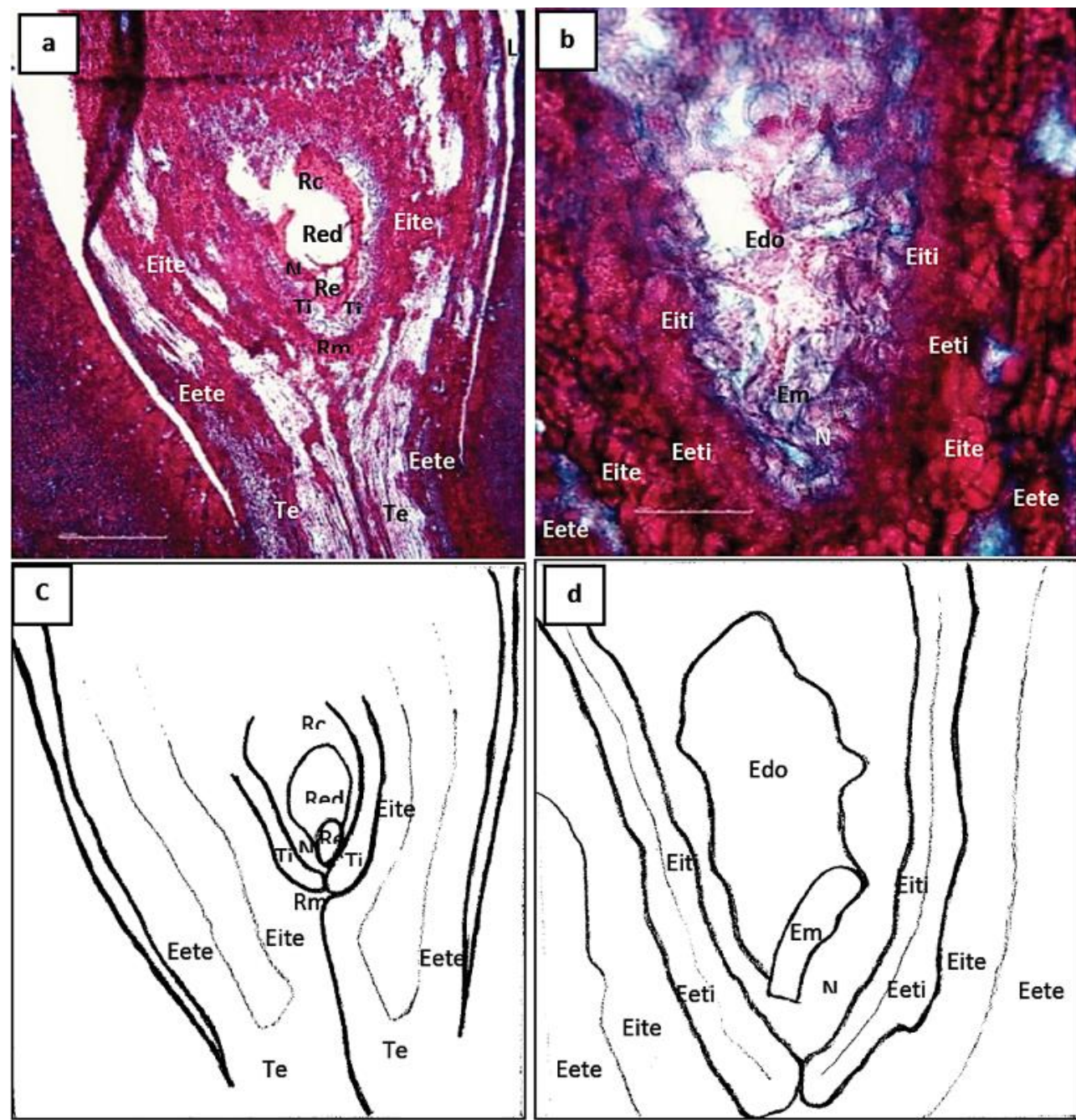

Figura 6. Desarrollo fenológico del fruto de N. lappaceum a los 26 DDA, en a) corte longitudinal del fruto, en b) inicio de la endospermogénesis y embriogénesis, en c) y d) representaciones esquemáticas. Edo: endospermo, Eete: epidermis externa del tegumento externo, Eeti: epidermis externa del tegumento interno, Eite: epidermis interna del tegumento externo, Eiti: epidermis interna del tegumento interno, Em: embrión, N: nucela, Rc: región calazal, Re: región del embrión, Red: región del endospermo, Rm: región micropilar, Te: tegumento externo, Ti: tegumento interno. Escala: $\mathrm{a}=50 \mu \mathrm{m}, \mathrm{b}=100 \mu \mathrm{m}$

Uno de los indicadores más importantes en la madurez del fruto, fue el cambio a color rojo intenso del pericarpio incluyendo los espiternos (Reyes et al., 2020). El pericarpio es seco y fácil distinguir a simple vista sus partes anatómicas derivadas del carpelo (Arévalo et al., 2018). El tejido vascular se observó en la región media del pericarpio, su localización está relacionada con la forma en que los carpelos se unen (Nacif et al., 2001). 
El fruto presenta un pericarpio seco, indehiscente y monospermo. Proviene de un gineceo bicarpelar en el cual, hacia la madurez solo se desarrolla un carpelo y el otro se atrofia, quedando rudimentario y unido al pedúnculo (Figura 7B), por lo que se trata de una nuez esquizocárpica (fruto indehiscente originado por un gineceo con dos o tres carpelos). Este resultado coincide con el estudio de Pham et al. (2015) para D. longan y con el de Nacif et al. (2001) para L. chinensis, quienes mencionaron que solo un óvulo se desarrolla en fruto hacia la madurez.

Los espiternos presentan una epidermis multiestratificada, se empiezan a desarrollar a los 15 ó 20 días después de la caída de pétalos, se encuentran unidos al pericarpio hasta la maduración (Figura 9B). Son más gruesos hacia su parte basal y miden de $1 \mathrm{a} 1,5 \mathrm{~cm}$ de longitud, son suaves y quebradizos, existe una conexión vascular entre el pericarpio y los espiternos, los cuales son responsables de la deshidratación del fruto ya que tienen hasta 250 estomas por $\mathrm{mm}^{2}$ presentando una alta transpiración (Kothagoda y Rao, 2012). Cambian de color verde a rojo en la madurez comercial, y dos semanas más tarde, el color del pericarpio se vuelve rojo intenso (Figura 7A y 9A).

Cuadro 2. Derivados gametofíticos y tegumentarios del óvulo, en la semilla de N. lappaceum

\begin{tabular}{ll}
\hline \multicolumn{1}{c}{ Óvulo } & \multicolumn{1}{c}{ Semilla madura } \\
\hline Saco embrionario & Embrión \\
Rudimento seminal & Endospermo \\
Parénquima nucelar & Semilla \\
Epidermis nucelar & Se desorganiza \\
Epidermis interna del tegumento interno & Se desorganiza \\
Parénquima interepidérmico del tegumento interno & Endotegmen \\
Epidermis externa del tegumento interno & Mesotegmen \\
Epidermis interna del tegumento externo & Exotegmen \\
Parénquima interepidérmico del tegumento externo & Endotesta \\
Epidermis externa del tegumento externo & Mesotesta \\
Funículo & Exotesta \\
\hline
\end{tabular}

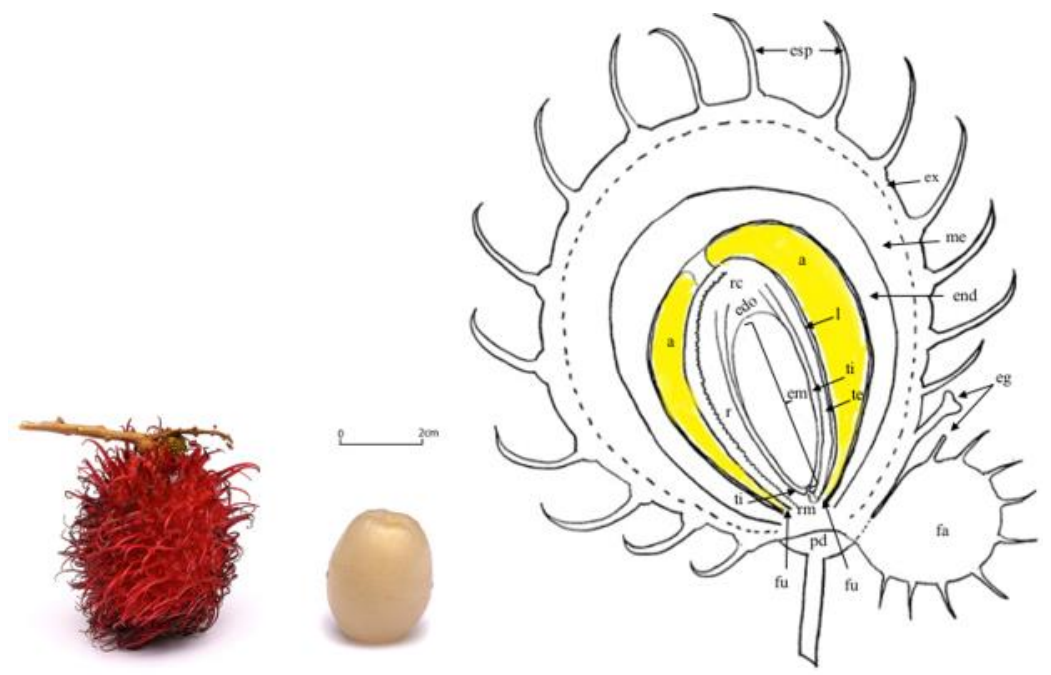

Figura 7. Estructura del fruto de N. lappaceum a los 70 DDA; en A) fruto con y sin pericarpio, en B) estructura del fruto conteniendo a la semilla. a: arilo, edo: endospermo, eg: estigma, em: embrión, end: endocarpio, esp: espiterno, ex: exocarpio, fa: fruto abortivo, fu: funículo, l: lóculo, me: mesocarpio, pd: pedúnculo, r: rafe, rc: región calazal, rm: región micropilar, te: tegumento externo, ti: tegumento interno 

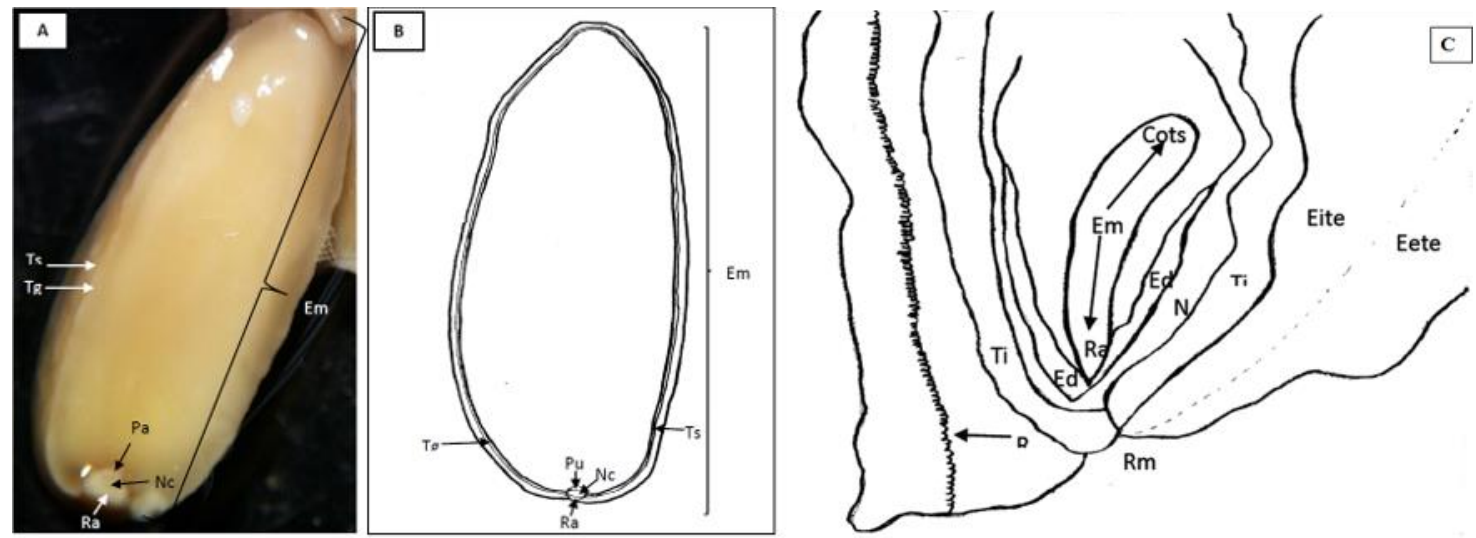

Figura 8. Anatomía de la semilla de N. lappaceum, en A) corte longitudinal en semilla madura a los 125 DDA, en B) representación esquemática de semilla madura, en C) representación esquemática de semilla joven a los 25 DDA. Cots: cotiledones, Ed: endospermo, Eete: epidermis externa del tegumento externo, Eite: epidermis interna del tegumento externo, Em: embrión, N: nucela, Nc: nudo cotiledonal, Pa: plúmula, Ra: radícula, Rm: región micropilar, Tg: tegmen, Ti: tegumento interno, Ts: testa. Escala: $50 \mu \mathrm{m}$

El arilo constituye la parte comestible, que deriva de la base del funículo de la semilla y comienza a formarse a los 70 DDA, cubriéndola en su totalidad hacia la madurez. Presenta 1,5 $\mathrm{cm}$ de grosor en promedio, está formado por células alargadas radialmente, a menudo muy grandes y de paredes primarias engrosadas; contiene un jugo de sabor azucarado muy agradable. Este es el último tejido en terminar de desarrollarse en el fruto (Figura 9A). Contrariamente, Kothagoda y Rao (2012) afirman que el arilo en el fruto de $N$. lappaceum se origina por la división del tegumento interno del óvulo, y lo consideran una baya.
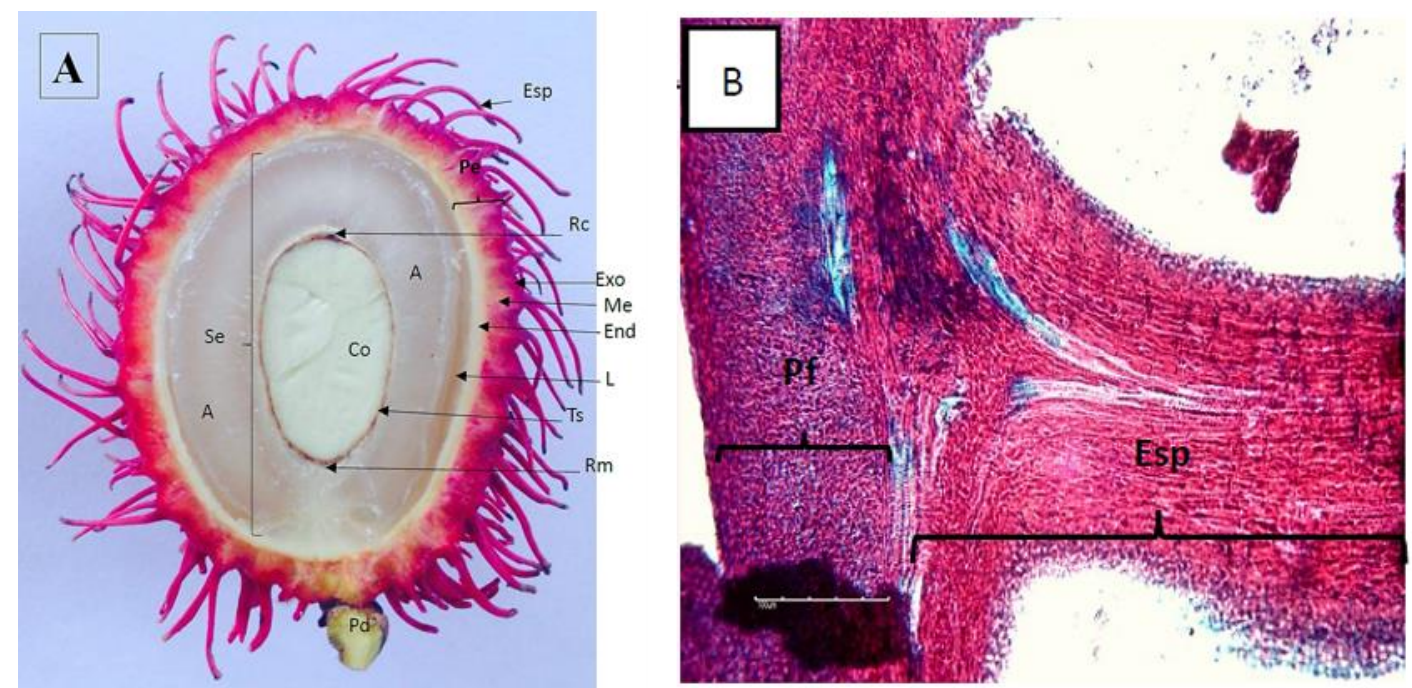

Figura 9. Fruto maduro de N. lappaceum, cortes longitudinales; en A) vista general a los 125 DDA, en B) anatomía del espiterno y pared del fruto. A: arilo, Co: cotiledón, End: endocarpio, Esp: espiternos, Exo: exocarpio, L: lóculo, Me: mesocarpio, Pd: pedúnculo, Pe: pericarpio, Pf: pared del fruto, Rc: región calazal, Rm: región micropilar, Se: semilla, Ts: testa

El porcentaje de frutos inmaduros y abortados, hasta la fecha de maduración fue muy alto $(91 \%)$. El muestreo de la infrutescencia con aquellos frutos que iniciaron su formación y desarrollo, se presentó a los 16 DDA, fecha en la que ya había ocurrido la polinización. Es probable que la caída de frutos con 
diámetro menor a $1 \mathrm{~cm}$ sea consecuencia de una debilidad en la pared celular en la zona de abscisión del pedúnculo, fenómeno relacionado con un desbalance fitohormonal (relación auxinas-etileno) y nutrimental en las hojas antes de la floración (Thitithanakul, 2016).

Resultados similares fueron reportados por Djuita et al. (2016), quienes obtuvieron hasta un 98 $\%$ de frutos caídos en árboles de $N$. lappaceum. En L. chinensis se sugiere que la caída de flores y frutos también puede deberse a que durante la antesis el desarrollo vegetativo se reanuda, por lo que, durante este periodo los órganos vegetativos y reproductivos presumiblemente compiten por cantidades limitadas de fotoasimilados (Stern y Gazit, 2003).

\section{CONCLUSIONES}

La iniciación floral en $N$. lappaceum se presentó a los 42 DDA luego de un estrés hídrico; los primeros cambios que se observaron en el meristemo apical, fueron el aplanamiento del domo meristemático y la elongación de la inflorescencia meristemática. La flor hermafrodita presentó un fenómeno identificado como separación carpelar postgénita, con un gineceo de tipo esquizógeno. El fruto, ovoide y con crecimiento sigmoidal simple, alcanzó su máximo tamaño y color, a los 125 DDA. El pericarpio del fruto fue seco, indehiscente, monospermo, derivado de un carpelo (nuez esquizocárpica) de color rojo. La semilla fue arilada y exalbuminosa, con el tegumento externo más grueso que el interno y con el rafe que entra hasta la región calazal. El arilo comenzó su desarrollo 70 DDA, se derivó del funículo de la semilla y fue el último tejido del fruto en desarrollarse.

\section{LITERATURA CITADA}

1. Arévalo-Galarza, M.L., J.F. Caballero-Pérez, G. Valdovinos-Ponce, J. Cadena-Iñiguez y C.H. Avendaño-Arrazate. 2018. Growth and histological development of the fruit pericarp in rambutan (Nephelium lappaceum Linn.). Acta Horticulturae 1194: 165-172.

2. Arias, C.M., R.H. Velásquez, C.D. Mateus, Z.H. Chaparro y R.J. Orduz. 2016. El rambután (Nephelium lappaceum), frutal asiático con potencial para Colombia: avances de la investigación en el piedemonte del Meta.
Revista Colombiana de Ciencias Hortícolas 10(2): 262-272.

3. Barrell, P.J. y U. Grossniklaus. 2005. Confocal microscopy of whole ovules for analysis of reproductive development: the elongate 1 mutant affects meiosis II. The Plant Journal 43(2): 309-320.

4. Baroux, C., C. Spillane y U. Grossniklaus. 2002. Evolutionary origins of the endosperm in flowering plants. Genome Biology 3(9): 1-5.

5. Caballero-Pérez, J.F., L. Arévalo-Galarza, C.H. Avendaño-Arrazate, J. Cadena-Iñiguez, G. Valdovinos-Ponce y J.F. Aguirre-Medina. 2011. Cambios físicos y bioquímicos durante el desarrollo y senescencia de frutos de rambután (Nephelium lappaceum L.). Revista Chapingo Serie Horticultura 17(1): 31-38.

6. Cao, L.M., N.H. Xia y Y.F. Deng. 2008. Embryology of Handeliodendron bodinieri (Sapindaceae) and its systematic value: development of male and female gametophytes. Plant Systematics and Evolution 274(1): 17-23.

7. Djuita, N.R., A. Hartana, T. Chikmawati y D. Dorly. 2016. Pulasan [(Nephelium ramboutanake (Labill.) Leenh.] fruit trees: variations in flower morphology, and associated differences in pollination type. International Journal of Plant Biology 7(1): 1-6.

8. Endress, P.K. 2011. Angiosperm ovules: diversity, development, evolution. Annals of Botany 107(9): 1465-1489.

9. García, E. 2004. Modificaciones al Sistema de Clasificación Climática de Köppen. Instituto de Geografía, Universidad Nacional Autónoma de México, México. 93 p.

10.González, V.V., S.M. Solís y M.S. Ferrucci. 2014. Anatomía reproductiva en flores estaminadas y pistiladas de Allophylus edulis (Sapindaceae). Boletín de la Sociedad Argentina de Botánica 49(2): 207-216.

11.Haseloff, J. 2003. Old botanical techniques for new microscopes. BioTechniques 34(6): 11741182.

12.Johri, B.M., K.B. Ambegaokar y P.S. Srivastava. 2014. Comparative embryology of angiosperms. Springer-Verlag Berlín Heidelberg, Alemania. 1221 p.

13.Joo-Pérez, R., C.H. Avendaño-Arrazate, A. 
Sandoval-Esquivez, S. Espinoza-Zaragoza, M. Alonso-Báez, J.L. Moreno-Martínez, R. ArizaFlores y C.R. Morales-Nieto. 2017. Alternancy study on rambutan (Nephelium lappaceum L.) tree in Mexico. American Journal of Plant Sciences 8(1): 40-52.

14.Kothagoda, N. y A.N. Rao. 2012. Fruit anatomy of four Nephelium species-with special reference to aril development. Journal of Tropical Medicinal Plants 13(2): 199-212.

15.Lim, A.L. 1984. The reproductive biology of rambutan, Nephelium lappaceum L. (Sapindaceae). Gardens' Bulletin, Singapore 37(2): 181-192.

16.Li-Min, C. y X. Nian-He. 2009. Floral organogenesis of Delavaya toxocarpa (Sapindaceae; Sapindales). Journal of Systematics and Evolution 47(3): 237-244.

17.Menzel, C. 2001. The physiology of growth and cropping in lychee. Acta Horticulturae 12(558): 175-184.

18. Muhamed, S., S. Kurien, K. Iyer, A. Remzeena $\mathrm{y}$ S. Thomas. 2019. Natural diversity of rambutan (Nephelium lappaceum L.) in Kerala, India. Genetic Resources and Crop Evolution 66(4): 1073-1090.

19.Nacif, S.R., P.A. Sartori y S.L. Chamhum. 2001. Morphological and anatomical development of the litchi fruit (Litchi chinensis Sonn. cv. Brewster). Fruits 56(4): 225-233.

20.Pham, V.T., M. Herrero y J.I. Hormaza. 2015. Phenological growth stages of longan (Dimocarpus longan) according to the $\mathrm{BBCH}$ scale. Scientia Horticulturae 189: 201-207.

21.Reyes-Moreno, M., E. García-Villanueva, S. Chávez-Franco, A. Muratalla-Lúa, J. ReyesMoreno y J.M.E. Aguilar-Luna. 2020. Fertilización en rambután (Nephelium lappaceum L.) para mejorar la calidad de frutos.
Ecosistemas y Recursos Agropecuarios 7(1): e2412.

22.Sandoval, Z.E. 2005. Técnicas Aplicadas al Estudio de la Anatomía Vegetal. Instituto de Biología, UNAM, México. 277 p.

23.Shields, V.D.C. y T. Heinbockel. 2018. Introductory Chapter: Histological Microtechniques. In: Histology. Heinbockel T. (ed.). IntechOpen. pp: 3-16.

24.Skinner, D.J. y V. Sundaresan 2018. Recent advances in understanding female gametophyte development [version 1; peer review: 2 approved]. F1000Research 7 (F1000 Faculty Rev): 804.

25.Stern, R.A. y S. Gazit. 2003. The reproductive biology of the lychee. Horticultural Reviews 28: 393-453.

26. Thitithanakul, S. 2016. Nutrients in leaves before flowering of rambutan (Nephelium lappaceum L.) cv. RongRien. Songklanakarin Journal of Scienc e and Technology 3(1): 40-44.

27.Weckerle, C.S. y R. Rutishauser. 2005. Gynoecium, fruit and seed structure of Paullinieae (Sapindaceae). Botanical Journal of the Linnean Society 147(2):159-189.

28.Windarsih, G. y M. Efendi. 2019. Morphological characteristics of flower and fruit in several rambutan (Nephelium lappaceum) cultivars in Serang City, Banten, Indonesia Biodiversitas 20(5): 1442-1449.

29. Yadegari, R. y G.N. Drews. 2004. Female gametophyte development. Plant Cell 16: S133S141.

30.Zini, M.L., G.B. Galati, S.M. Solís y M.S. Ferrucci. 2012. Another structure and pollen development in Melicoccus lepidopetalus (Sapindaceae): An evolutionary approach to dioecy in the family. Flora 207(10): 712-720. 